\title{
NEW COASTAL VIDEO-MONITORING SYSTEM ACHIEVEMENT AND DEVELOPMENT
}

\author{
Nico Valentini ${ }^{1}$, Leonardo Damiani ${ }^{1}$, Matteo Gianluca Molfetta ${ }^{1}$ and Alessandra Saponieri ${ }^{1}$
}

A new coastal video monitoring system for Apulia region, southeast of Italy, is under development. It is composed of visible and thermal streams and featured to be included in a wider meteo-oceanographic monitoring network. The system is designed for use on sandy beaches. The regional Basin Authority (AdBP) through previous field campaigns has identified for long-term monitoring purposes two hotspots, prone to erosion and flooding: Torre Canne (Fasano, BR) and Torre Lapillo (Porto Cesareo, LE), facing the Adriatic and Ionian seas, respectively. This paper presents the actual architecture and some initial findings of its implementation aiming at a complete automatic analysis of morphological features and hydrodynamic studies, mainly focused in swash zone.

Keywords: coastal video monitoring; run-up modelling; coastal management

\section{INTRODUCTION}

Since the late 1970s, erosion phenomena are considered among the major processes which deplete the coasts of the Earth. This acts as a huge concern for public authorities working for environmental resources protection, nowadays related also to sea level rise studies and relative consequences.

Moreover, a growing alarmism for inundation risk perception and the problem of loss of the beaches grew are considered to heighten the economic impact for the tourism industry (Phillips and Jones, 2006).

Large part of research community believes in sea level rise as the driving phenomenon of erosion processes (e.g. Leatherman et al., 2000; Zhang et al., 2004). Whereas many other causes contribute at small/medium spatial scales. Specifically, in Apulia region, the huge influence in the medium-term erosion processes has been demonstrated to be due to mutation in extreme storm surges, great changes of sediments supply, human-induced practices (i.e. inefficacy of groins and outfall protections, poseidonia banquettes removal), tectonic subsidence, etc. (Delle Rose, M., 2015; Regione Puglia - POR 2000-2006, 2009).

In this context, coastal management detains a great importance, especially for places with large sea resources, in order to model the economic and social goods. A well-structured and continuous coastal monitoring programme implemented within a farsighted management strategy is required to support priority coastal interventions, aimed at the coastline protection or the mitigation of the erosive tendencies. Indeed, to accomplish such an evidently powerful scope, coastal monitoring and operating surveys have been established to represent a priority stage, in order to collect data useful for the study of hydrodynamic processes and morphological evolution, as well as defense or mitigation works effects and efficacy. Monitoring facilitates well-informed decision-making and strategic planning for management purposes.

During the last two decades, video systems application has acquired a great confidence among the remote sensing monitoring techniques. The frequency inadequacy of measurement of shoreline position and its time variability, using i.e. cross-shore profiles surveys and aerial photographs, makes the landbased video platforms and the digital image-processing techniques optimal instruments to derive objective shoreline evolution over time. The reliability, accuracy and versatility of the coastal video systems have been tested and reviewed in scientific literature (Davidson et al., 2007).

Since the first technique was introduced to remotely measure scales and morphology of natural sand bars (Lippmann and Holman, 1989), algorithms for shoreline detection and beach slope estimation (Aarninkhof et al., 2003; Damiani, L. and Molfetta, MG., 2008, Plant and Holman, 1997) have been successfully accomplished. Moreover, the use of terrestrial camera platforms has been extended within the context of wave empirical and statistical parameterization of run-up (Holland et al., 1995; Stockdon et al., 2006), nearshore bathymetries and data-assimilation for forecasting analysis (Aarninkhof et al., 2005; Holman et al., 2013; van Dongeren et al., 2008), swimming safety and current predictions (Archetti and Romagnoli, 2011; Radermacher et al., 2014), management of traditional and soft defense systems (Saponieri and Damiani, 2015) and coastal state indicators deployment (Jimenez et al., 2007; Kroon et al., 2007).

In the Apulia region, the application of such a system is still at initial stages of development, mainly because of the relative high cost of commercial products that has made the littoral video-monitoring difficult to be deployed and maintained.

For this reason, the development of a new, efficient, almost completely automatic and low-cost system is fully justified. This has been achieved through the development of a video monitoring and

\footnotetext{
${ }^{1}$ DICATECh, Technical University of Bari, via Orabona 4, 70125 Bari, Italy
} 
surveillance system, built upon calibrated non-metric IP surveillance cameras, able to accurately quantify coastal processes.

The system has been designed to be included in the broader Apulian meteo-oceanographic monitoring network, which is active since 2006 and currently managed by the local regional Apulian Basin Authority (AdBP) (Damiani L. et al, 2007). This monitoring network includes, to date 2 wave buoys, 4 tide stations , 6 anemometric stations and 2 new web-cam stations. Its collected data are stored in a control center in real time via GSM, freely available on the web portal http://simopadb.dyndns.org/web/simop/misure.

Furthermore, within this context, a new specific model for shoreline detection (Shoreline Detection Model, SDM), has been developed. The SDM calibration and validation has been performed on coastal images derived from a video monitoring system installed back at Alimini (Lecce, IT) in 2005, by comparing automatic shoreline contours with manual detected ones, on several cross-shore transects.

The main objective of this paper is to show the structure and development of the new system and present initial findings. The paper is structured in 2 main parts: (i) the main architecture of the system; (ii) the SDM model and its first morphological and hydrodynamic applications.

\section{New Apulian Video Monitoring System}

The new video monitoring system has been built through a close cooperation between the Technical University of Bari, DICATECh department, Multitel research center, Image Processing R\&D department (http://www.multitel.be/) and its spin-off ACIC (http://www.acic-tech.be/). The system development aimed at some core factors including easy installation, robustness, low cost, efficiency of the acquisition and tasks scheduling. In order to meet these objectives, few firm principles are taken into account: (i) to disjoin the post-processing tasks from the acquisition ones (the former take place on cloud computing server, the latter take place on-site and enable the independence on type of cameras and hardware solutions from post-processing steps); (ii) to find a compromise between compact and cost-effective, network cameras solution; (iii) to guarantee all the calibration procedures, including the lens distortion correction, to be applied on-site, without affecting overall accuracy; (iv) to contribute to the distribution of video-analysis results by building an user-friendly, Java based, interface in order to gain a broad based support and public participation.

The image acquisition procedure has been implemented by using state of the art autonomous IP cameras, detached from archiving/processing server or laptop, which guarantee a decentralized framework; hence, the acquisition phase could be preserved during components maintenance, by using substituted hardware. The redundancy ensured by the storage on both local low-cost servers running Unix based operating system and remote servers is a key factor in the system deployment. By doing this, one could choice best solution for local Hard-Drive copy, while the web-portal is served by server storage via rest call.

The stream of each camera installed is configured in such a way to collect video records of $10 \mathrm{~min}$ and frequency of $1 \mathrm{~Hz}$. The sampling interval is $30 \mathrm{~min}$. The main products produced and stored into the local servers are three fundamental types of images: Snapshot, Timex and Variance and video (Timestack). The transfer to a cloud server is guaranteed by local router $4 \mathrm{G}$ connectivity. The remote postprocessing tasks are applied once each new image and data are uploaded. Specifically, the SDM model is applied on the Timex images. A sea level data filter is routinely executed, which produces the level used for geo-rectification procedure. This geo-rectification takes place after a $3 * 4$ perspective transformation matrix is opportunely defined. An iterative solver is used by minimizing the sum of the reprojection errors, which corresponds to the accumulated squared distances between the 3D point projection and its measured 2D coordinate. The minimization is applied by using Levenberg-Marquardt algorithm, for both internal and external calibration estimations. It shall be highlighted that aluminium foil tapes stuck on paper are properly used for building targets useful for thermal sensor calibration in order to enhance emissivity gradient (Figure 1).

The shoreline detected on the oblique image are geo-rectified as well. These tasks are executed and automatized by using Python scripting, partially including libraries from OpenCV, flamingo-openearth and Gdal/OSR. Figure 1, at the top, shows the targets used for internal and external calibrations of the cameras, at the bottom locations of the installed system, nearest tidal gauges and the approximate FoV of cameras. 


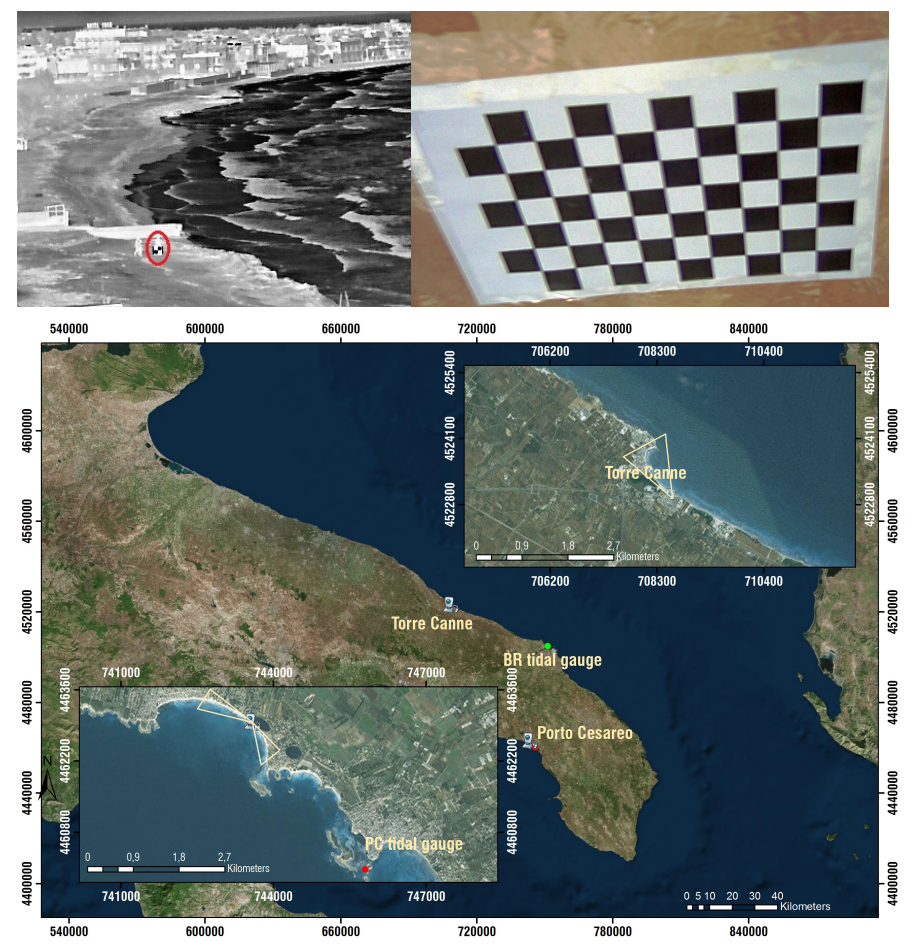

Figure 1. Top Left to right: Example of Pattern used as GCP to perform external and internal calibration procedure for thermal and visible camera, respectively; Bottom: New Video Monitoring station installed at Apulia region: Camera locations and approximate Field of View (FoV). Nearest tidal buoy (dots) of Apulia monitoring network, BR: Brindisi; PC: Porto Cesareo . Grid coordinates: RDN2008/TM33.

Moreover, in this context, a procedure useful in order to correct the perspective transformation matrix, $P$ after unexpected changes of the precise camera Centre of View $(\mathrm{CoV})$ location due to atmospherically factors or other accidents, is developed. It has been demonstrated to be extremely important in order to reduce these significant geo-rectification errors (Holman and Stanley, 2007, Voudoukas et al., 2011). It is based on SURF algorithm (Bay et al., 2008), geometry transform estimator with RANSAC schema (Fischler and Bolles, 1981) and Procrustes analysis (Kendall, 1989). Estimated accuracy after an ad-hoc correction is overall below 0.4 pixel for PC shore. The correction is going to be yield automatic. A sum-up of main tasks implemented in new coastal video monitoring system is described in Figure 3.

\section{Web-tool}

The possibility of sharing the on-going and final results of coastal video stations is quite promising, supporting professionals and coastal managers in morphological and hydrodynamic analysis. Moreover, by exploiting resource users or stakeholders' involvements, through sharing them pre and post-processed results, help instilling the concept of so-called participatory monitoring. Indeed, people including members of the communities who live in the vicinity of the monitored zone, but also who make use of the resources (i.e. hoteliers, drive operators, tourists, SCUBA divers) may be aware on actual beach state and its evolution, being encouraged to increase a feeling of "ownership" of the ecosystems and motivated to protect and conserve them. This has led to the construction of a web-portal (http://91.121.30.84), which includes the publication of results in a quasi-real time (Figure 3). The web-site is specifically built by using JavaScript and coded HTML, and it is a 1.0 version, released and included into the main AdBP coastal web-area (http://simopadb.dyndns.org/web/simop).

It is developed as a five modules web-page for different consultation tabs. The first tab is intended to list the last images produced at all the installed stations of the system, among those produced (Snap, Timex, STD and geo-rectified); the Images Report allows user to view thumbnails and download images, choosing interval and extremes date of a time period. Shoreline map tab permits a mapping of the georectified shoreline, as dots, over the rectified geo-referenced image, while in order to perform cross-shore multi-temporal analysis Shoreline Transect Analysis is a tool able to plot the shoreline distances to a baseline over time, after a cross-shore transect is chosen, from a displayed sequence over an oblique 
frame (Figure 4). The Download tab allow users to download all the data produced by the monitoring stations. In Figure 2, few screenshots of the web-tool are presented.

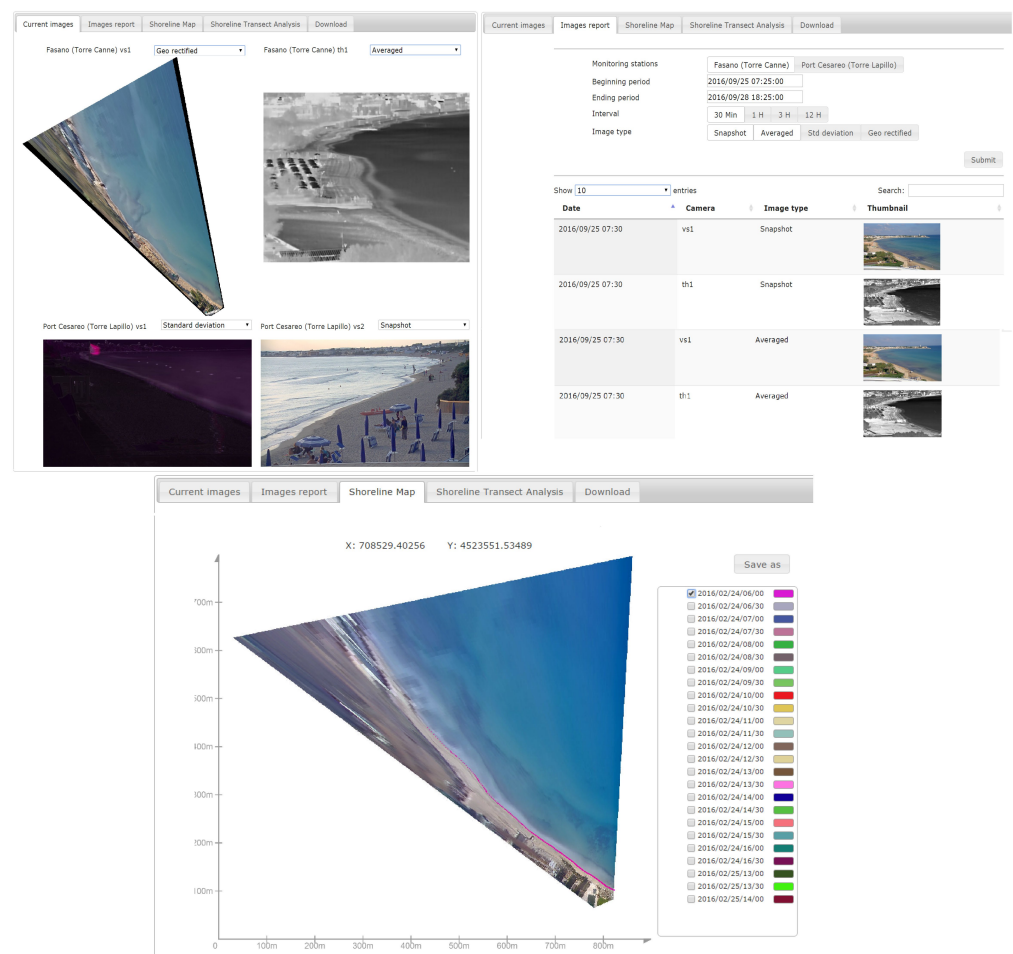

Figure 2. Top Left to right: Current Images and Images Report tabs. Bottom: Shoreline Map tab of the system web-tool.

\section{System Implementation and Study area}

The Apulian regional Basin Authority (AdBP) through previous field campaigns has identified for long-term monitoring purposes two hotspots, prone to erosion and flooding: Torre Canne (Fasano, Br) and Torre Lapillo (Porto Cesareo, LE). At Torre Canne (TC) site two network cameras are installed on a pole, which is fixed on parapet of an Hotel (708616.1 E, $4522941.0 \mathrm{~N}$ ). The approximate orthometric height of the cameras is $23.4 \mathrm{~m}$ (ITALGEO2005).

The station consists of one thermal $\left(T C_{t h}\right)$ and one visible $\left(T C_{v s}\right)$ cameras, both faced $N N W$, with a Field of View (FoV) of $10^{\circ}$ and $59.5^{\circ}$, respectively. An Uncooled Micro bolometer $384 \times 288$ sensor features the thermal cam, while the $T C_{v s}$ produces frame resolution with $1920 \times 1080$ pixel through HDTV 1080p video in multiple, individually configurable H.264 and Motion JPEG video streams and has a built-in IR illumination. Both are Axis ${ }^{\circledR}$ Cameras.

The coastal area of TC, facing the Adriatic Sea, represents an important thermal location for Apulia region. It is constituted by several free and private beaches characterized by medium grain size sand, according to Udden-Wentworth scale, with a mean diameter of the particle size distribution, $D_{50}$ and a $D_{95}$ equal to $0.28 \mathrm{~mm}$ and $0.44 \mathrm{~mm}$, respectively. The emerged beach is about $20 \div 50 \mathrm{~m}$ wide, characterized by a steep berm with periodically vegetation encroachments. The wave climate in the area is moderate, with an annual significant wave height $\left(H_{s}\right)$ ranging from $0.5 \mathrm{~m}$ to $1.5 \mathrm{~m}$ for the $49.75 \%$ of wave observations in $1990-2007$. The $0.16 \%$ of the measured waves heights exceeds $3.5 \mathrm{~m}$. The $67.6 \%$ of observations is a peak period from 3 to $7 s$ constitutes. The analysis of wave climate shows that waves approach from $N N W$ for the $29.7 \%$ of the time, while a discrete contribute of the sector ESE occurs for around $18 \%$ (Petrillo A.F. and LIC, 2010).

The great urbanization rate, mainly due to touristic activities, has been crucial in increasing the negative sedimentary balance of the area (AdB-Puglia, 2015). Indeed, in the last decade, the analysis of long-term shoreline movements, highlights a global erosional trend, with maximum shoreline withdraws equal to about $10 \mathrm{~m}$ at few spots, which, has become quite stable in recent years. 


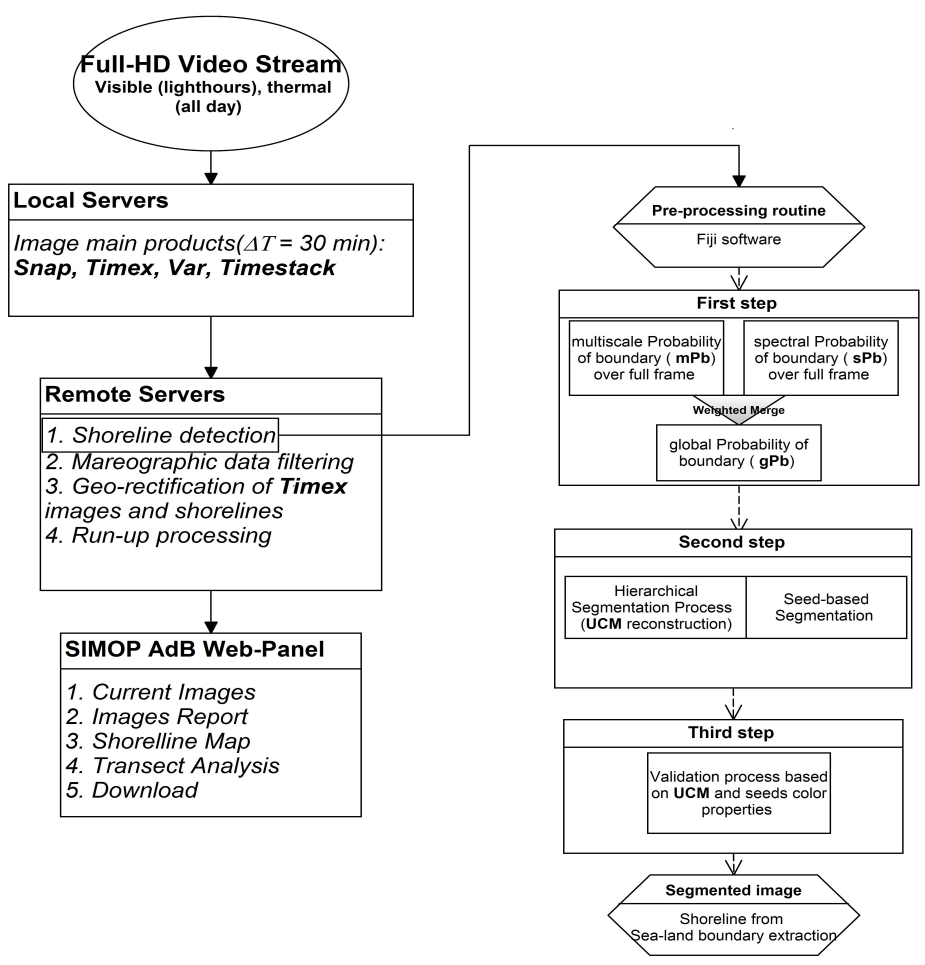

Figure 3. Process outline of main tasks of new coastal video monitoring system and sum-up of algorithms, which build the SDM.

The video-station placed at Torre Lapillo, hamlet of Porto Cesareo (PC) (743584.0 E, $4462960.2 \mathrm{~N}$ ) includes a couple of identical visible cameras: the first one stared at $N W$ direction, $P C_{v s 1}$, with FoV equal to $51.6^{\circ}$, while the second one, $P C_{v s 2}$, faced to $S E$, with a FoV of $56.2^{\circ}$. Both are Axis model as in TC installation.

The Marine Area of Porto Cesareo is an interesting site from a morphological point of view. This area is constituted by typical sub-environment of low-lying coasts, with calcarenitic rocky and sandy beaches. Specifically, the large embayed beach of Torre Lapillo, is characterized by a typical asymmetric planform shape, featured by quite strongly curved zone (at $N W$ ), a gently curved centre (where video station is installed) and a relative straight section (at $S E$ ). Sandy beaches are characterized by a mean diameter $D_{50}$ almost equal to $0.47 \mathrm{~mm}$ and $D_{95}$ to $1.38 \mathrm{~mm}$. Since the first 60 's, the number of establishments has been increased in the geographical area of Porto Cesareo, with remarkable damages of emerged beach, especially of dunes. The establishments' managers apply a common defense practice during the winter season, by means of geotextile sandbags which are uniformly distributed just few meters close to coastline, determining huge morphological changes in the ordinary and stormy berms trying to prevent great shore erosion.

A large erosional trend in the period 2009-2011 has been recognized, and mainly attributed to the mean sea level rise (around $10 \mathrm{~cm}$ ), amplified by significant storms occurred (AdB-Puglia, 2015). Particularly, the shoreline erosional hotpots are located in southeast, where in 2013-2015 the cumulative sediments balance resulted less than $-10 \mathrm{~m}$. For the majority of the time $(49.7 \%)$ the waves approach from $S S E$, while for $34.8 \%$ they come from $S W$ direction.

The wave climate in the area is moderate to low, with an annual significant wave height less than $0.75 \mathrm{~m}$ for the $44 \%$ of observations in 2006-2013, while a $12.17 \%$ for $0.75<H_{s}<1.75 \mathrm{~m}$ and only the $0.66 \%$ with $H_{s} \geq 3.0 \mathrm{~m}$ (AdB-Puglia, 2015). The peak period $T_{p}$ in the interval $3 s$ to $5 \mathrm{~s}$ represents the most frequent (31.9\% around).

Coastal vulnerability assessment in these areas is of great interest because of the tourism economy of this coastal stretch, which represents a great resource for local community. 


\section{System applications}

\section{Shoreline Detection Model}

The new SDM, briefly described hereafter, is the results of the boundary extraction from automatic segmented coastal area; it has been calibrated and tested on the products of a previous video system, active in Apulia region, at Alimini (Le) shore specifically, between 2005-2009 (Damiani L. and Molfetta MG., 2008), and now implemented and tested on the new system, introduced above.

Time-averaged images are used as inputs. With respect to other models, widely adopted in literature (e.g. Aarninkhof et al., 2003; Kingston, 2003; Osorio et al., 2012), the perceptually uniform colour-space, the CIELab, is used for processing. A pre-processing routine is properly developed in order to bypass issues due to variation in illumination and contrast, mainly and filter out outliers (algae, objects, etc.). A distribution of the popular software ImageJ: Fiji (Schindelin et al., 2012), is chosen for this preprocessing task. Fiji is one of the most used software in biological field for generating advanced imageprocessing pipelines, it guarantees processing large quantities of images via scripting languages and feature-rich libraries, based on the simplicity of Java.

The histograms of the masked image by two small seed areas (around 200 pixels each) representative of the sea and sand pixel surfaces are calculated; this framed seeds are supposed to remain constant during long-term monitoring. Specifically, a normalized Chi-Square histogram (Pele and Werman, 2010) distance, $H_{d}$ on these seed surfaces, is assumed to determine the best contrasting color feature, between $L$ and $b$ color channels, useful for subsequent processes.

The pre-processing steps include mainly contrast enhancement, median filtering, bi-exponential edge-preserving smoother (BEEPS), which is basically a version of the bi-exponential edge preserving smoother with adaptive weights (Thévenaz et al., 2012) applied on the best contrasting color channel, $L$ or $b$ of CIELab. On the pre-processed images, the main routine for automatic segmentation and then, shoreline detection algorithm, is applied. A subdivision of the main routine can be summarized as follows: i) contours detector algorithm over the full frame; ii) 2-steps segmentation; iii) intertidal bar solver and correction process. The first step is basically a modified version global Probability of boundary $(g P b)$ concept of Arbelaez et al., 2011. It is thought in order to combine the benefits of bottomup techniques, such as simple edge detection, with those of top-down approaches like clustering, including both approaches into a probabilistic framework.

The 2-steps segmentation tasks follow the boundaries detection process. The algorithms employed reported in (Arbelaez, 2006, Arbeláez and Cohen, 2008, Maire, 2009), are implemented in order to produce a hierarchical boundaries map (Ultrametric Contour Map, UCM) that becomes useful for enforcing regions closure and which is used for a subsequent seed-based segmentation, allowing few framed overall distinct areas of the shore to be classified. Then, front propagation strategy on the UCM map by using Voronoi tesselations is applied. Inputs are the UCM and a set of seed pixels, in this implementation, they are labelled as Sea water, Sand and Sky. The outputs are constituted by the ultrametric distances of a general pixel from seeds and the relative labels.

The last process stage of the main routine has been thought in order to search for intertidal bars boundaries and correct the results of the previous constrained segmentation, using the colour properties of the UCM regions, based on $H_{d}$.

The SDM is calibrated and tested on the products of a video system previously installed in Alimini (Le). The validation process derived by the distance calculation, in pixel units, between manual and automatic shoreline contours at several cross-shore transects.

Hence, the SDM is tested with successfully results on both study areas. The shorelines contours are computed in a completely automatic task. In particular, Figure 4 refers to a 5 months-long times-series of shoreline evolution at TC, extracted on a single cross-shore transect. It is compared to the time-series of the significant wave height measured by the nearest wave gauge of the AdBP network. Results highlighted the high correlation of the two time-series.

A longer investigation interval will allow further insights on beach morphological evolution.

\section{Comparison between measured and numerical predicted run-up}

The new video system is also employed for semi-automatic run-up measurements. Wave run-up processes are not easy to measure, particularly under extreme conditions. Powerful wave forces and significant beach changes can damage observing equipment or introduce uncertainty in the underlying topographic elevations needed to understand the run-up processes. Two approaches useful to circumvent data collection challenges could be based on ground-remote observations and on numerically simulations of swash zone and run-up. 

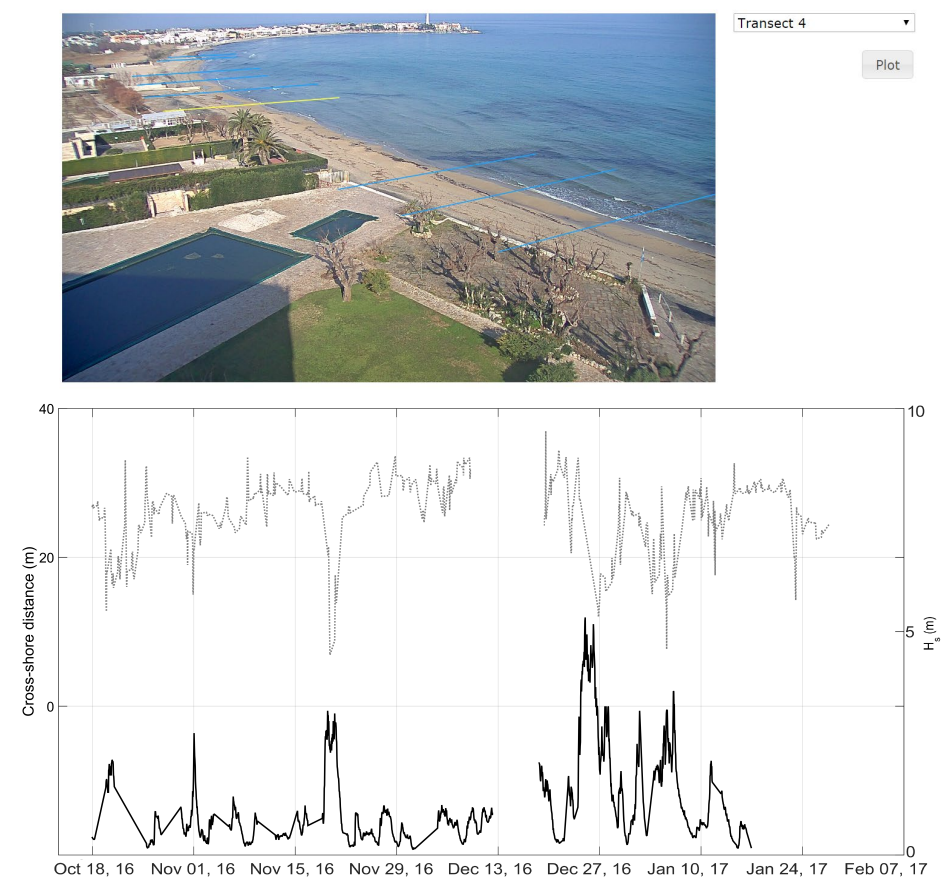

Figure 4. Top: Screenshot extracted from the "Shoreline transect analysis" tab of the web-tool and refers to the $4^{\text {th }}$ cross-shore transect, user-selected (yellow line); Bottom: time series of shoreline distance calculated from baseline (black) compared with significant wave height recorded by nearest wave gauge of AdBP network (dashed grey).

An analysis using 10 cross-shore transects at PC shore, almost equally distributed every $10 \mathrm{~m}$, by processing time-stack images is here presented. Real Time Kinematic surveys using differential GPS solution on the Global Navigation Satellite System (GNSS) of Apulia Region were carried out on 1st March 2016, supported by the AdBP personnel. Recorded video has a duration of 30 min and the acquisition frequency used is $5 \mathrm{~Hz}$.

A routine for time-stack processing before applying any method for swash extremes recognition is developed, in order to deal with dirty and altered pixels due to geo-textiles bags, algae of a nearest beach establishments. It includes manual masking of the mentioned pixel areas, background subtraction, BEEPS and variance filters on the grey coded images. The Otsu thresholding method, also employed in Vousdoukas et al., 2011, is used for extracting swash maxima. The obtained pixel values, expressing cross-shore coordinates of swash extremes, are transformed into elevations using topographical information, run-up time series are calculated, statistical exceedance values indeed used for comparison with numerical results: $R_{2}$ and $R_{\max }$.

A novel numerical methodology is implemented, which combines a wide validated forecasting dataset, MeteOcean (Mentaschi et al., 2015), with nested SWAN and SWASH (Zijlema et al., 2011) models in order to achieve accurate and computationally feasible simulation of waves at different time and spatial scales, up to the total energy dissipation in the swash zone. SWASH is chosen since in literature it is revealed to be numerically more straightforward with respect to similar phase resolving model (i.e. Bousinnesq wave model MIKE21 BW), in terms of numerical robustness (De Roo et al., 2015), easiness in implementation and open source orientation.

The SWASH simulations are performed in 1-d domains along the cross-shore transects where topographical measurements are collected. A step of $0.05 \mathrm{~m}$ is used, with mean length of around $700 \mathrm{~m}$. In the present study, the Manning's roughness coefficient $n$ is used, as the bottom friction formulation. The default configuration setting for this parameter in SWASH is equal to $n=0.019 \mathrm{~s} / \mathrm{m}^{1 / 3}$. Due to the difficulties in measuring it and not univocal results in literature about its optimal value, it could represent a useful and straightforward fitting parameter for numerical model calibration. In the present study, a sensitivity analysis of the SWASH model capability in predicting hydrodynamics, by varying the Manning's roughness factor, ranging from 0.01 to $0.04 \mathrm{~s} / \mathrm{m}^{1 / 3}$, according to the most typical field relevant values, is reported.

Summarizing, the bottom friction reveals a significant influence on wave run-up, and the value of $n=0.04 \mathrm{~s} / \mathrm{m}^{1 / 3}$ exhibits the best fit in predicting the time-stack measurements, as highlighted in 
Figure 5. Specifically, with respect to $R_{2}$ calculation the $R M S E \cong 0.075 \mathrm{~m}$, while for $R_{\max }$, the $R M S E \cong 0.062 \mathrm{~m}$. The methodology proposed and presented in this paper can be used as a practical tool for identification of the potential run-up excursion, exceedance values and if the case, extreme flooding analysis at the embayment of Torre Lapillo, thus providing a valuable information for coastal authorities, to be also extended on the coasts of Apulia region, affected by wide erosion phenomena.
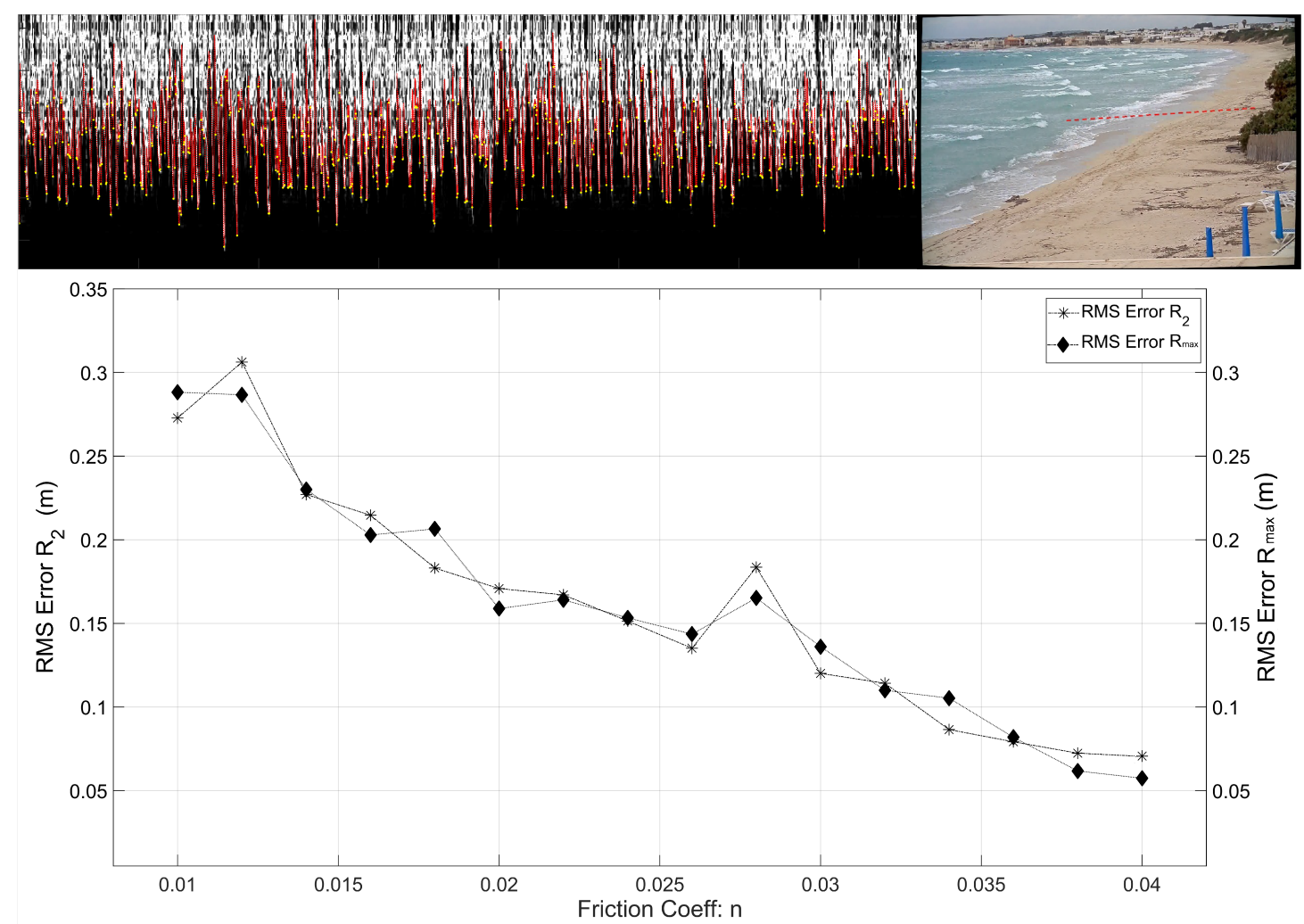

Figure 5. Top Left to right: Time-stack with the y-axis that represents the cross-shore transect location of video-derived run-up observations and the $\mathrm{x}$-axis the time variability $(20 \mathrm{~min})$. Camera $P C_{v s 2}$ undistorted FoV and one oblique-view transect (red dashed line) highlighted. Bottom: Sensitivity of bottom friction with respect to computed $R_{2}$ and $R_{\max }$.

\section{CONCLUSIONS}

In the paper, a new video coastal video monitoring system deployed and integrated in the Apulia Region Monitoring Network (South Italy) is presented, and first results illustrated. In order to prevent irreversible erosion processes as well as flooding damages continuous studies and assessment of the coastal states play a fundamental role. In this context, the video monitoring represents a low-cost instrument to store a large amount of data, due to the feasibility in extracting several morphodynamic and hydrodynamic measurements from images/videos. The automatic analysis yielded on remote server guarantees low involvement of coastal experts over time and sharing results via web-tool keep involved community in risk perception and facilitate professionals and coastal managers in morphological and hydrodynamic analysis. With respect to the numerical analysis of run-up, it must be highlighted that by varying the friction Manning coefficient can have a significant effect on the 1-d SWASH modelled runup and so, on the extents of inundation. If SWASH is used for estimating real-world hazards, users should think carefully about choosing an appropriate value, and may want to run sensitivity studies. In this study, the 1-d SWASH results are satisfying with an ad-hoc calibration of the Manning value of the friction coefficient and $n=0.04 \mathrm{~s} / \mathrm{m}^{1 / 3}$ results to best fit the time-stack measurements.

Thermal sensor will be further used for main purposes: comparing the efficacy in shoreline detection with respect to visible camera, calibration of new supervised methods for classification of different beach areas and sediment sources evaluation, moreover image processing at night-light hours would guarantee following severe storms evolution and extreme events monitoring. 


\section{ACKNOWLEDGMENTS}

This research is partially supported by Authority of Basin of Apulia Region, AdBP (Italy), particularly in the persons of Prof. Antonio Di Santo (general Secretary), Dott. Nicola Palumbo, and Dott. Roberto Francioso. We thank our colleagues from ACIC (http://www.acic-tech.be) who provided expertise in web-tool creation and management.

\section{REFERENCES}

Aarninkhof, S. G., Turner, I. L., Dronkers, T. D., Caljouw, M. \& Nipius, L. 2003. A video-based technique for mapping intertidal beach bathymetry. Coastal Engineering, 49, 275-289.

Aarninkhof, S. G. J., Ruessink, B. G., \& Roelvink, J. A. (2005). Nearshore subtidal bathymetry from time-exposure video images. Journal of Geophysical Research: Oceans, 110.

AdB-Puglia. 2015. Monitoraggio delle dinamiche meteomarine di controllo dei fenomeni di erosione delle coste. Technical Report from AdBP.

Arbelaez, P. Boundary extraction in natural images using ultrametric contour maps. Computer Vision and Pattern Recognition Workshop, 2006. CVPRW'06. Conference on, 2006. IEEE, 182-182.

Arbelaez, P., Maire, M., Fowlkes, C. \& Malik, J. 2011. Contour detection and hierarchical image segmentation. Pattern Analysis and Machine Intelligence, IEEE Transactions on, 33, 898-916.

Archetti, R., Romagnoli, C., 2011. Analysis of the effects of different storm events on shoreline dynamics of an artificially embayed beach. Earth Surface Processes and Landforms 36, 1449-1463.

Bay, H., Ess, A., Tuytelaars, T. \& Van Gool, L. 2008. Speeded-up robust features (SURF). Computer vision and image understanding, 110, 346-359.

Damiani L., Bruno M. F., Molfetta M. G., Nobile B. 2007. Coastal zone monitoring in Apulia region: first analysis on meteomarine climate. ISEH V 2007 Fifth International Symposium for Environmental Hydraulics - Arizona 2007.

Damiani, L., \& Molfetta, M. G. (2008). A video based technique for shoreline monitoring in Alimini (LE). In Coastlab08 (pp. 153-156). L. Damiani, M. Mossa.

Davidson, M., Van Koningsveld, M., de Kruif, A., Rawson, J., Holman, R., Lamberti, A., Medina, R., Kroon, A. \& Aarninkhof, S. 2007. The CoastView project: Developing video-derived Coastal State Indicators in support of coastal zone management. Coastal Engineering, 54, 463-475.

De Roo, S., Suzuki, T., Kolokythos, G., Zhao, G. \& Verwaest, T. Numerical modelling of 2D wave transformation processes from nearshore to a shallow foreshore: comparison between the Mike21, swash and XBeach models. 36th IAHR World Congress, 2015. 1-6.

Delle Rose, M. (2015). Medium-term Erosion Processes of South Adriatic Beaches (Apulia, Italy): A Challenge for an Integrated Coastal Zone Management. J Earth Sci Clim Change, 6(306), 2.

Fischler, M.A., Bolles, R.C., 1981. Random sample consensus: a paradigm for model fitting with applications to image analysis and automated cartography. Communications of the ACM 24, 381395.

Holland, K. T., Raubenheimer, B., Guza, R. T., \& Holman, R. A. 1995. Runup kinematics on a natural beach. Journal of Geophysical Research: Oceans, 100 (C3), 4985-4993.

Holman, R., Plant, N. \& Holland, T. 2013. cBathy: A robust algorithm for estimating nearshore bathymetry. Journal of Geophysical Research: Oceans, 118, 2595- 2609.

Jimenez, J.A., Osorio, A., Marino-Tapia, I., Davidson, M., Medina, R., Kroon, A., Archetti, R., Ciavola, P., Aamikhof, S.G.J., 2007. Beach recreation planning using video-derived coastal state indicators. Coastal Engineering 54, 507-521.

Kendall, D.G., 1989. A survey of the statistical theory of shape. Statistical Science, 87-99.

Kroon, A., Davidson, M.A., Aarninkhof, S.G.J., Archetti, R., Armaroli, C., Gonzalez, M., Medri, S., Osorio, A., Aagaard, T., Holman, R.A., Spanhoff, R., 2007. Application of remote sensing video systems to coastline management problems. Coastal Engineering 54, 493-505.

Leatherman, S. P., Zhang, K., \& Douglas, B. C. 2000. Sea level rise shown to drive coastal erosion. Eos, Transactions American Geophysical Union, 81(6), 55-57.

Lippmann, T. and Holman, R. A. 1989. Quantification of sand bar morphology: a video technique based on wave dissipation. Journal of Geophysical Research: Oceans (1978-2012), 94, 995-1011.

Maire, M. 2009. Contour detection and Image Segmentation. Dissertation, University of California, Berkeley.

Mentaschi, L., Besio, G., Cassola, F. \& Mazzino, A. 2015. Performance evaluation of Wavewatch III in the Mediterranean Sea. Ocean Modelling, 90, 82-94.

Pele, O., \& Werman, M. 2010. The quadratic-chi histogram distance family. In European conference on computer vision (pp. 749-762). Springer Berlin Heidelberg. 
Petrillo, A. F. and LIC 2010. Determinazione del clima meteomarino al largo e sottocosta e del trasporto solido per paraggi significativi della costa pugliese. Report, Bari: DIAC.

Plant, N. G. \& Holman, R. A. 1997. Intertidal beach profile estimation using video images. Marine Geology, 140, 1-24.

Phillips MR, Jones AL. 2006. Erosion and tourism infrastructure in the coastal zone: Problems, consequences and management. Tourism Management 27: 517-524.

Radermacher, M., Wengrove, M., Van Thiel de Vries, J., Holman, R., 2014. Applicability of videoderived bathymetry estimates to nearshore current model predictions, Proceedings of the 13th International Coastal Symposium. Journal of Coastal Research, Special Issue 70, 2014.

Regione Puglia - POR 2000-2006. 2009. Progetto di monitoraggio degli interventi di difesa costiera e dell'evoluzione dei litorali (- Misura 1.3, area di azione 2, sottoazione 2B, - Misura 1.3, ara di azione 4, sottoazione 4C). Technical Report of Apulian Region.

Saponieri, A., Damiani, L., 2015. Numerical analysis of infiltration in a drained beach. International Journal of Sustainable Development and Planning 10, 467-486.

Schindelin, J., Arganda-Carreras, I., Frise, E., Kaynig, V., Longair, M., Pietzsch, T., Preibisch, S., Rueden, C., Saalfeld, S., Schmid, B., 2012. Fiji: an open-source platform for biological-image analysis. Nature methods 9, 676-682.

Stockdon, H. F., Holman, R. A., Howd, P. A. \& Sallenger Jr, A. H. 2006. Empirical parameterization of setup, swash, and runup. Coastal Engineering, 53, 573- 588.

Thévenaz, P., Sage, D. \& Unser, M. 2012. Bi-exponential edge-preserving smoother. Image Processing, IEEE Transactions on, 21, 3924-3936.

Van Dongeren, A., Plant, N., Cohen, A., Roelvink, D., Haller, M. C., \& Catalán, P. 2008. Beach Wizard: Nearshore bathymetry estimation through assimilation of model computations and remote observations. Coastal engineering, 55(12), 1016-1027.

Vousdoukas, M., Ferreira, P., Almeida, L., Dodet, G., Psaros, F., Andriolo, U., Taborda, R., Silva, A., Ruano, A., Ferreira, Ó., 2011. Performance of intertidal topography video monitoring of a mesotidal reflective beach in South Portugal. Ocean Dynamics 61, 1521-1540.

Zhang, K., Douglas, B. C., \& Leatherman, S. P. 2004. Global warming and coastal erosion. Climatic Change, 64(1), 41-58.

Zijlema, M., Stelling, G., \& Smit, P. 2011. SWASH: An operational public domain code for simulating wave fields and rapidly varied flows in coastal waters. Coastal Engineering, 58(10), 992-1012. 